\title{
Theory of estimation-of-distribution algorithms
}

\author{
Witt, Carsten
}

Published in:

Proceedings of 2019 Genetic and Evolutionary Computation Conference

Link to article, DOI:

10.1145/3319619.3323367

Publication date:

2019

Document Version

Publisher's PDF, also known as Version of record

Link back to DTU Orbit

Citation (APA):

Witt, C. (2019). Theory of estimation-of-distribution algorithms. In Proceedings of 2019 Genetic and Evolutionary Computation Conference (pp. 1197-1225). Association for Computing Machinery.

https://doi.org/10.1145/3319619.3323367

\section{General rights}

Copyright and moral rights for the publications made accessible in the public portal are retained by the authors and/or other copyright owners and it is a condition of accessing publications that users recognise and abide by the legal requirements associated with these rights.

- Users may download and print one copy of any publication from the public portal for the purpose of private study or research.

- You may not further distribute the material or use it for any profit-making activity or commercial gain

- You may freely distribute the URL identifying the publication in the public portal

If you believe that this document breaches copyright please contact us providing details, and we will remove access to the work immediately and investigate your claim 
Theory of Estimation-of-Distribution Algorithms

\section{Carsten Witt}

DTU Compute

Technical University of Denmark

Tutorial at GECCO 2019

Updated version: https://tinyurl.com/eda2019

GECCO '19 Companion, July 13-17, 2019, Prague, Czech Republic.

( 2019 Copyright is held by the author/owner(s)

ACM ISBN 978-1-4503-6748-6/19/07

https://doi.org/10.1145/3319619.3323367
Carsten Witt is an associate professor at the Technical University of Denmark. He received his M. Sc. in 2000 and Ph. D. degree in 2004, both in Computer Science from the Technical University of Dortmund, Germany. His main expertise is in the algorithmic analysis of metaheuristics, including evolutionary algorithms, ant colony optimization and estimation-of-distribution algorithms. Carsten has over 80 peer-reviewed publications and has given tutorials about bio-inspired computation in combinatorial optimization at previous GECCO and PPSN conferences. He is member of the editorial boards of Evolutionary Computation Journal and

Theoretical Computer Science.

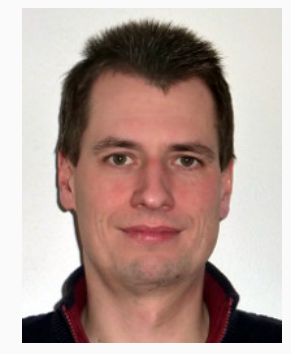

Overview

- Introduction to estimation-of-distribution algorithms (EDAs)

- What do we mean by theory?

- Presentation of important EDAs in theory

- Main results: from convergence to runtime analysis

- Conclusions

\section{Evolutionary Algorithms (EAs)}

- Work with populations of search points

- Modify the search points through, e. g., mutation and crossover

- Select promising search points based on fitness

\section{EDAs}

- Work with probability distributions

- Sample search points based on current distribution

- Adjust distribution based on the most promising search points 
An Estimation-OF-Distribution Algorithm
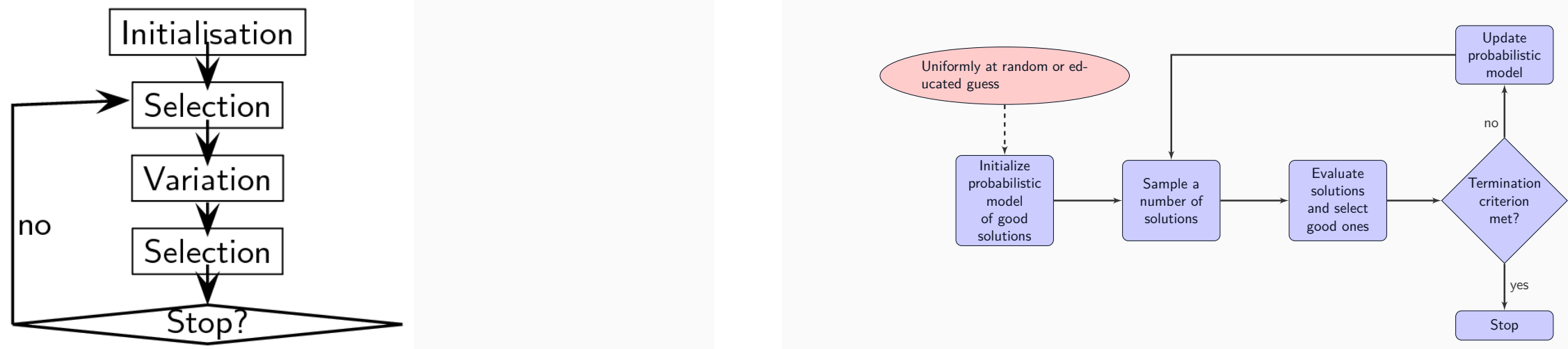

How an EA Approaches the Optimum

How an EA Approaches the Optimum
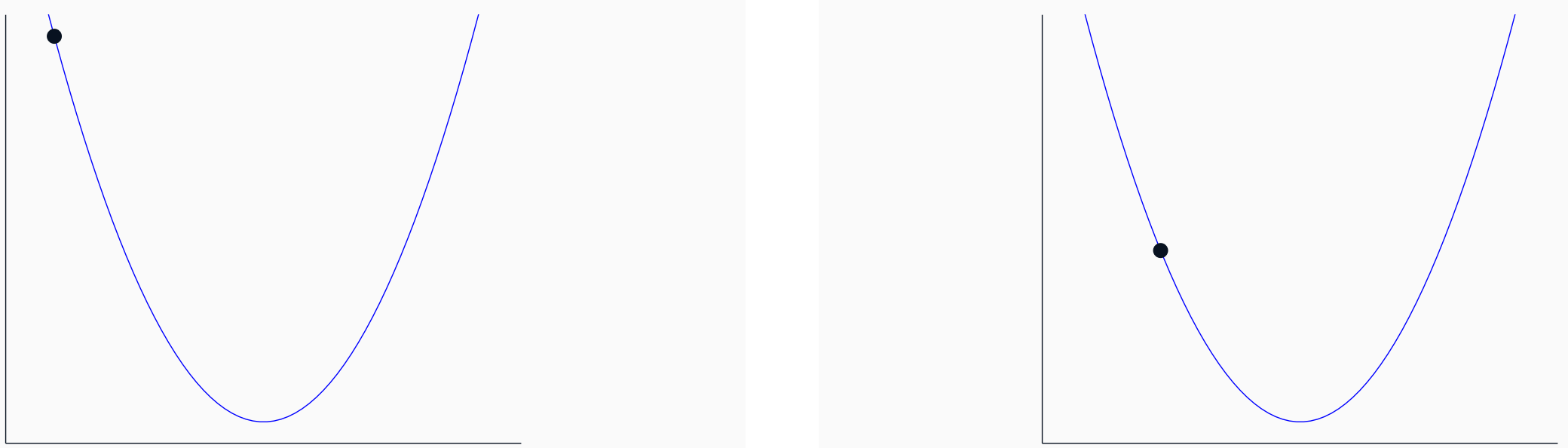

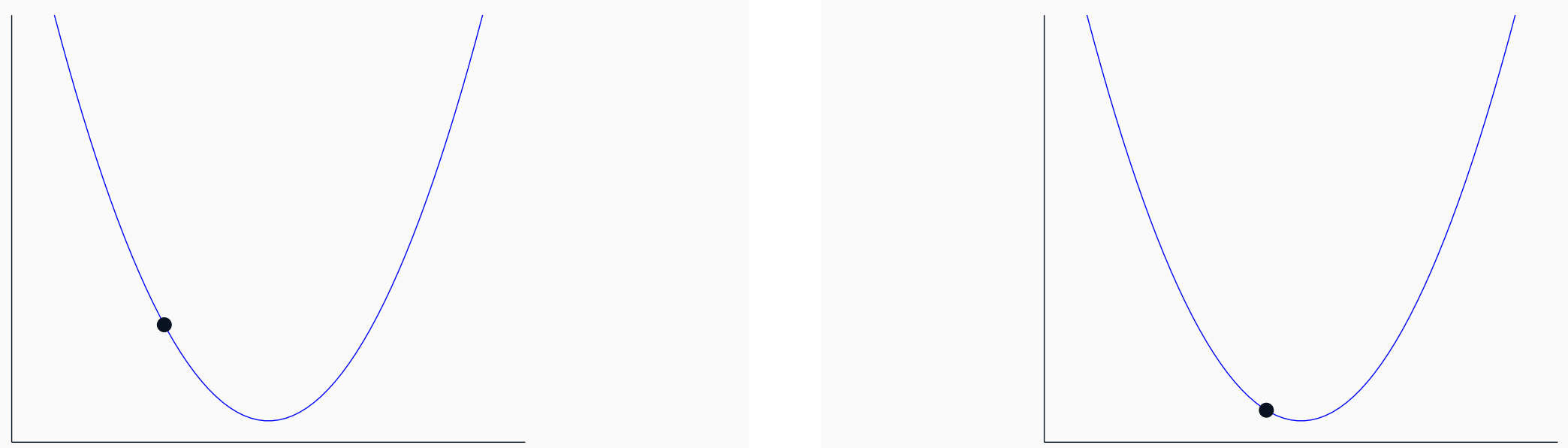

How an EA Approaches the Optimum

How an EDA Adjusts the Probabilistic Model
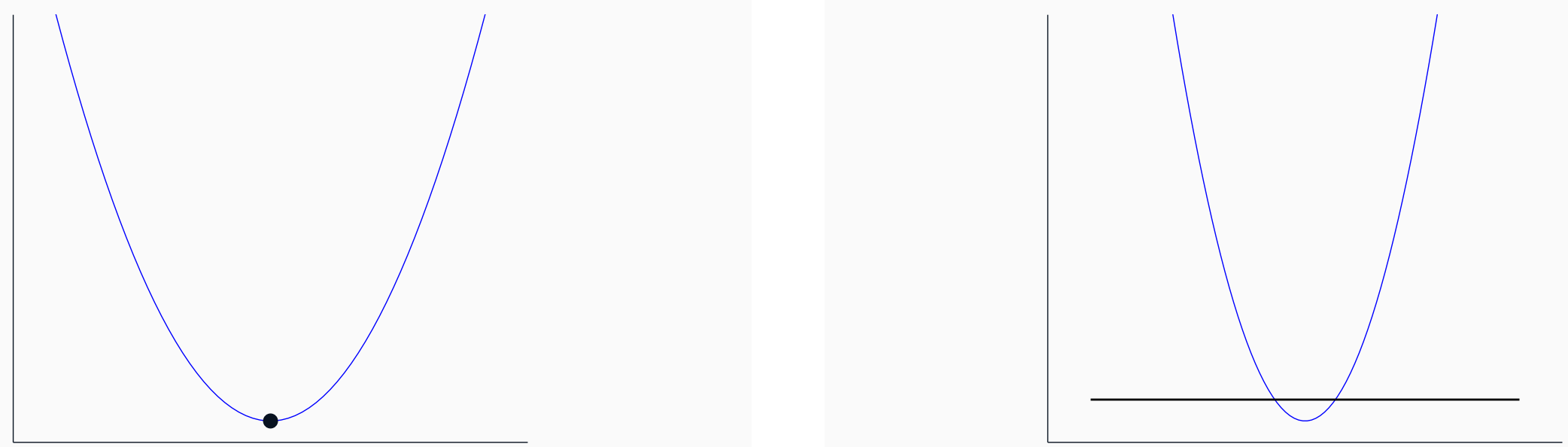

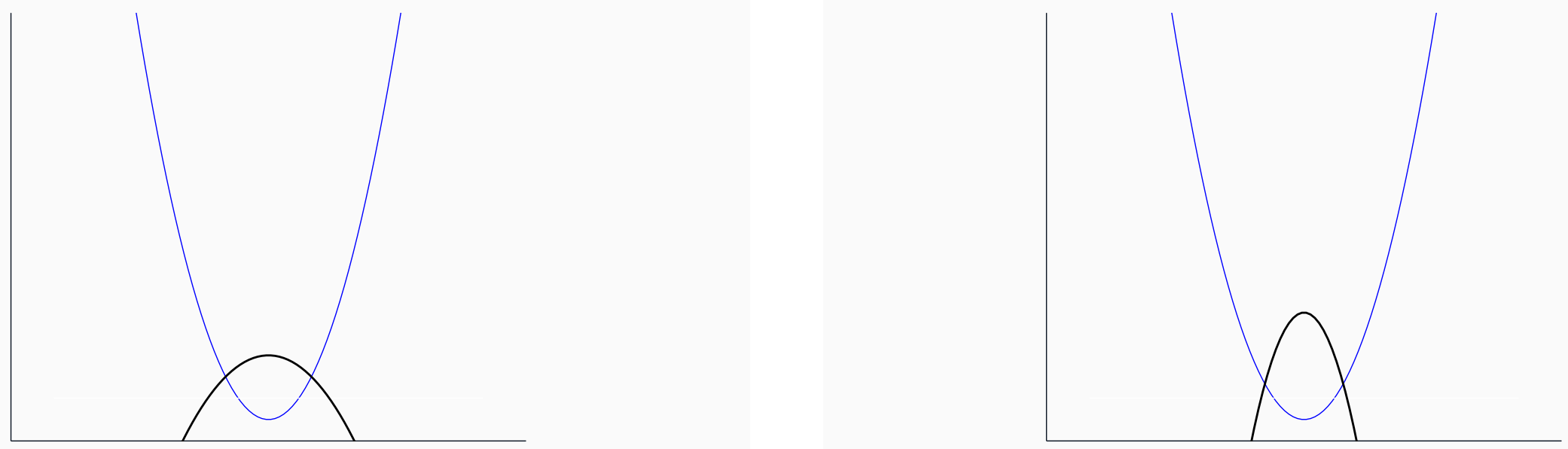

How an EDA Adjusts the Probabilistic Model
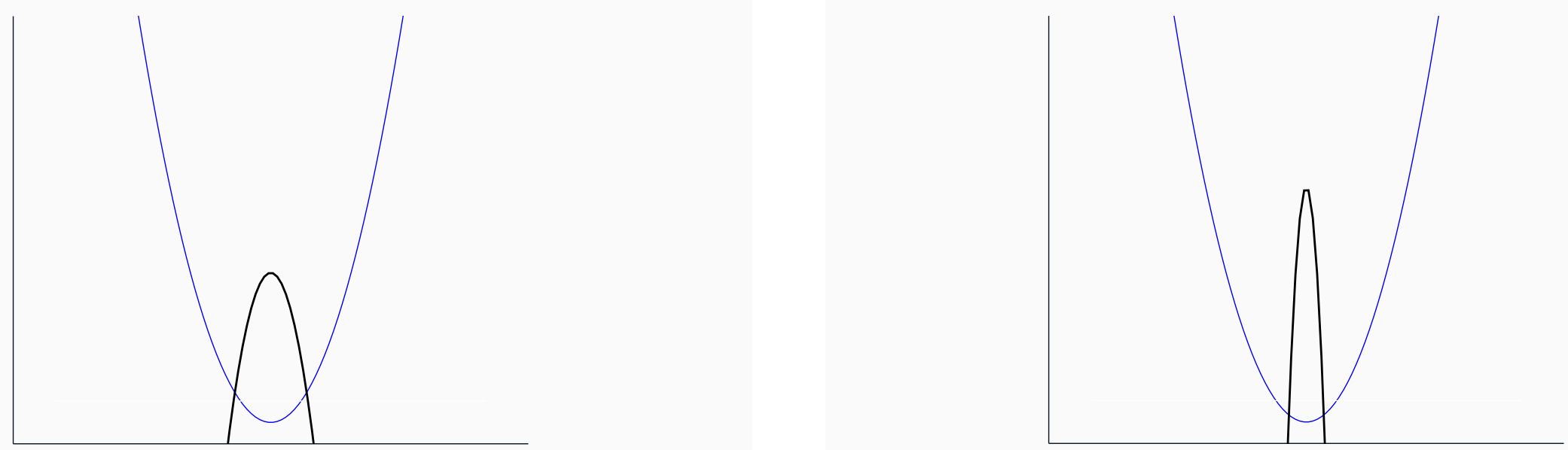


\section{CONTENTS}

IMPORTANT EDAS

Introduction

Preliminaries

OneMax

LeadingOnes

Binary Value

Noise

Jump

Stable EDAs

End
Domain: discrete optimization, e. g., find maximum for $f:\{0,1\}^{n} \rightarrow \mathbb{R}$. Important distinction: univariate vs. multivariate EDAs.

For example: $f\left(x_{1}, x_{2}, x_{3}\right)=-x_{1}+2 x_{1} x_{2}+x_{3}$. Good to learn learn dependency between $x_{1}$ and $x_{2}$.

Univariate

- cGA

- UMDA

- PBIL

- MMAS

$>\ldots$

\section{Multivariate}

$\checkmark$ FDA

- ECGA

- MIMIC, BMDA

- BOA

Most theoretical results concern univariate EDAs.

Some Benchmark Functions

Theoretical results often consider simple problems, which we have to understand first.

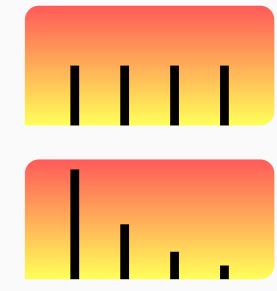

- $\operatorname{BinVaL}\left(x_{1}, \ldots, x_{n}\right):=\sum_{i=1}^{n} 2^{n-i} x_{i}$

111010101101

- LeAdingOnes $\left(x_{1}, \ldots, x_{n}\right):=\sum_{i=1}^{n} \prod_{j=1}^{i} x_{j}$

in more complex scenarios

Write OneMax but mean $n-\operatorname{Ham}(x, a)$ for unknown string $a \in\{0,1\}^{n}$.

Univariate Algorithms

Common concept: the frequency vector (aka. marginal probabilities)

$1-\frac{1}{n}$

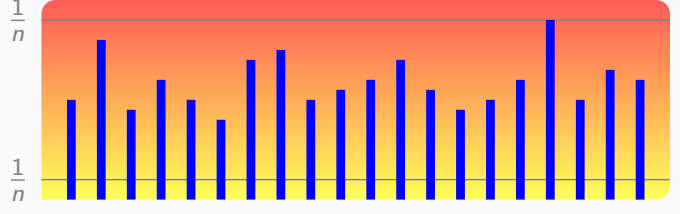

- Probabilities $\left(p_{1}, \ldots, p_{n}\right)$ for setting the individual bits to 1 .

- Usually initialized as $p_{i}=1 / 2$ for all $i$.

- Independently sampled.

- Frequency vector adjusted over time. 


\section{Compact GA (cGA) (HARIK et Al., 1999)}

Simulates behavior of a GA with population size $K$ in a compact way.

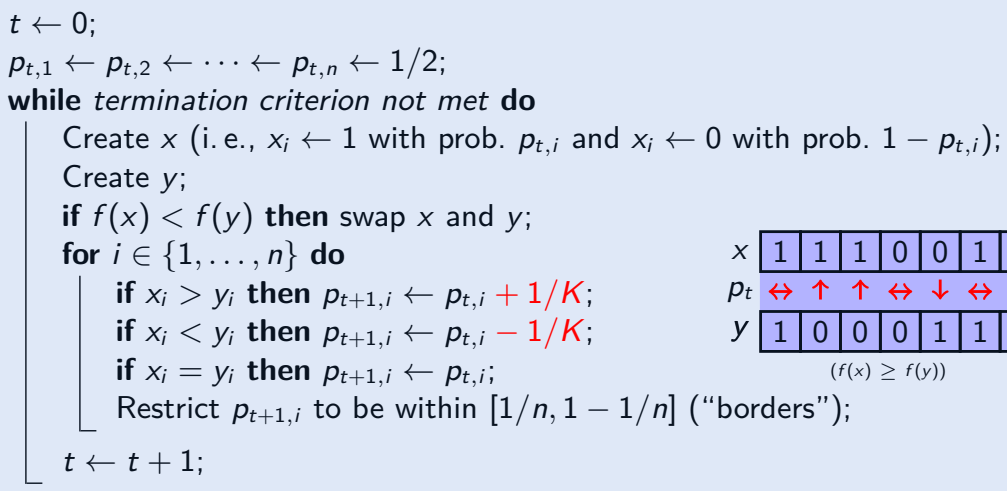

Parameter $K$ determines preciseness of model.

Big $K=$ fine model $=$ small update strength.
2-MMAS IB $_{\text {(NEUMAnN ET AL., 2010) }}$

Max-Min Ant System (Stützle and Hoos, 2000) with iteration-best update

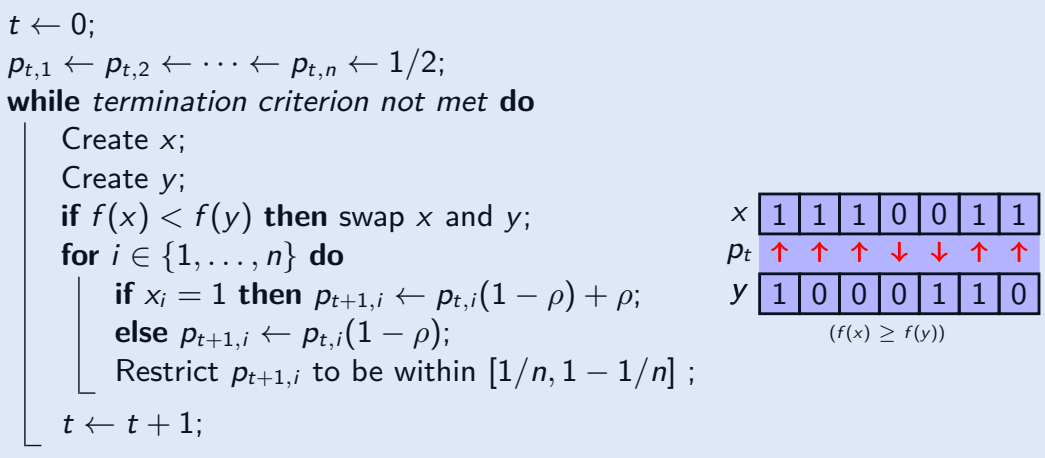

Here $1 / \rho$ reflects preciseness.

\section{UMDA (MÜHLEnBein ANd PAass, 1996)}

$t \leftarrow 0, p_{t, 1} \leftarrow p_{t, 2} \leftarrow \cdots \leftarrow p_{t, n} \leftarrow \frac{1}{2} ;$

while termination criterion not met do

$P_{t} \leftarrow \emptyset$;

for $j \in\{1, \ldots, \lambda\}$ do

for $i \in\{1, \ldots, n\}$ do

$x_{t, i}^{(j)} \leftarrow 1$ with prob. $p_{t, i}$

$x_{t, i}^{(j)} \leftarrow 0$ with prob. $1-p_{t, i} ;$

$P_{t} \leftarrow P_{t} \cup\left\{x_{t}^{(j)}\right\}$;

Sort individuals in $P$ descending by fitness (s.t.

$\left.f\left(x_{t}^{(1)}\right) \geq \cdots \geq f\left(x_{t}^{(\mu)}\right)\right)$, breaking ties u. a. r.;

for $i \in\{1, \ldots, n\}$ do

$p_{t+1, i} \leftarrow \frac{\sum_{j=1}^{\mu} x_{t, i}^{(j)}}{\mu} ;$

Restrict $p_{t+1, i}$ to be within $\left[\frac{1}{n}, 1-\frac{1}{n}\right]$;

$t \leftarrow t+1$;

If, e. g., $\mu=\lambda / 2$, then $\lambda$ reflects preciseness of model. 


\section{Genetic Drift}

If the fitness function is constant/flat (does not give a signal), frequencies move randomly to a border (DEMO).

$1-\frac{1}{n}$

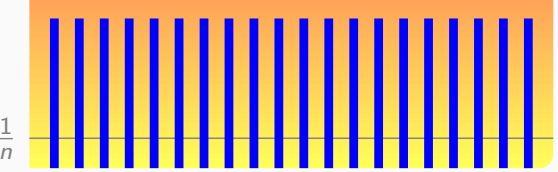

Frequencies that move to the wrong border are problematic - even disastrous if the border is not there.

Even if the expected value of a frequency converges to optimal value

(Höhfeld and Rudolph, 1997), this does not say much about runtime.

\section{CONTENTS}

Introduction

Preliminaries

OneMax

LeadingOnes

Binary Value

Noise

Jump

Stable EDAs

End

\section{Genetic Drift}

If the fitness function is constant/flat (does not give a signal), frequencies move randomly to a border (DEMO).

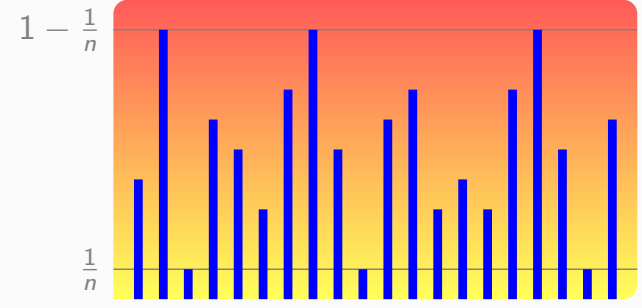

Frequencies that move to the wrong border are problematic - even disastrous if the border is not there.

Even if the expected value of a frequency converges to optimal value (Höhfeld and Rudolph, 1997), this does not say much about runtime.
EDAs, OneMaix

EARLY RESUlTS $(<2000)$

Models of cGA, UMDA and others, allowing estimations of the dynamical behavior (e. g., Thierens et al., 1998; Mühlenbein and Mahnig, 1999).

Two effects:

1. Overall progress of probabilistic model (roughly: $\left.\sum p_{i}\right)$ and time to convergence to good distribution $\left(\sum p_{i} \approx n\right)$,

2. time for single frequencies to drift to wrong border by genetic drift $\rightarrow$ should be bigger than convergence time.

Avoiding genetic drift requires precise enough model $\rightarrow$ lower bound on runtime. Models of EDAs estimated progress of frequencies:

$$
\begin{aligned}
& \text { Mühlenbein and Mahnig (1999) } \\
& \qquad p_{t+1, i} \approx p_{t, i}+\frac{l}{\sqrt{n}} \sqrt{p_{t, i}\left(1-p_{t, i}\right)},
\end{aligned}
$$

which was made rigorous recently. 
Genetic drift recurrent issue in analysis of GAs and EDAs, e. g., Asoh and Mühlenbein (1994); Shapiro $(2003,2005)$. Models proposed to make

frequencies "stable" (Friedrich et al., 2016a) $\rightarrow$ later

\section{Lower bound on preciseness of model}

For different EDAs on ONEMAX, same threshold identified multiple times (Thierens et al., 1998; Lobo et al., 2000; Shapiro, 2005): need at least $K=\Omega(\sqrt{n})$ different frequency values to prevent genetic drift.

\section{Upper bound on time to convergence}

Time for the EDA to converge to optimal solution $\approx K \sqrt{n}$

$\Rightarrow$ Best possible time complexity $\Theta(n)$ ?

All (?) analyses of convergence speed of EDAs before 2005 made some simplifying assumptions.

\section{Pros}

- Unimportant details ignored

- Insights possible that rigorous analyses cannot achieve

\section{Cons}

- No estimation of errors: verification by experiments

- No theorem: may only hold for small problem sizes

Since 2005: rigorous runtime analyses of EDAs, following the same principle as runtime analysis of EAs.
First RUNTIME ANALYSIS OF EDAs

Droste (2006) considered cGA on ONEMAX (and other functions)

Studied runtime $=$ no. iterations until optimum found.

\section{Main results}

- Upper bound $O(K \sqrt{n})$ on OneMax, for $K=\Omega\left(n^{1 / 2+\varepsilon}\right)$ $\rightarrow$ runtime $O\left(n^{1+\varepsilon}\right)$ (with high probability)

- General lower bound $\Omega(K \sqrt{n})$.

Recent refinement: Upper bound $O(n \log n)$ (Sudholt and W., 2016) for $K=c \sqrt{n} \log n$, big constant $c$

$\Rightarrow$ competitive with simple EAs (e. g., (1+1) EA).

Best lower bound until 2016: $\Omega(n)$ from general black-box complexity (Doerr and Lengler, 2015)

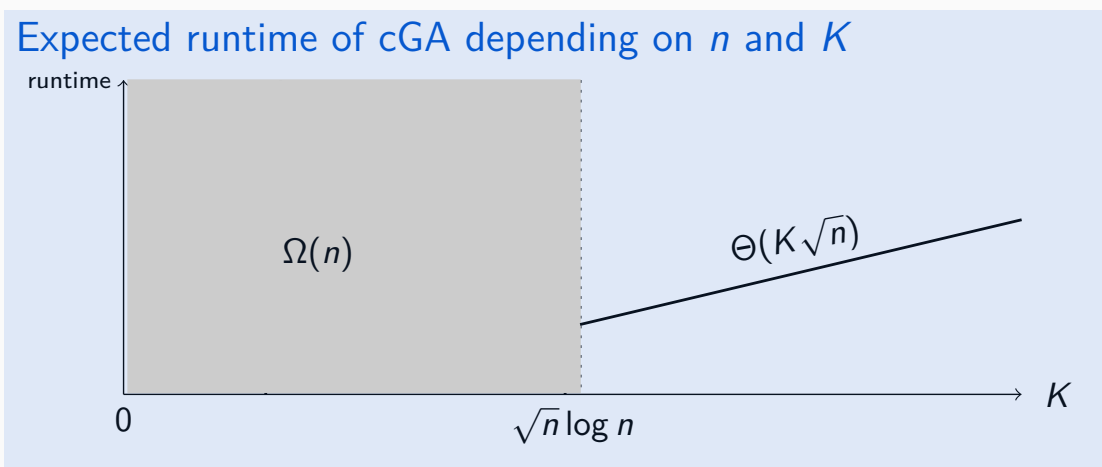


DYNAMICS ON BIT $i$

If $x_{i}=y_{i}$ then $p_{t, i}$ is unchanged.

If $x_{i} \neq y_{i}$ then $p_{t, i}$ is changed depending on $f(x)$ vs. $f(y)$ :

- Show limits on genetic drift: with high prob. all frequencies stay above $1 / 3$ in a phase of $\Theta(K \sqrt{n})$ steps.

- Frequencies move smoothly upwards (DEMO).

- To analyze speed: consider $\Phi_{t}=\sum_{i=1}^{n} p_{t, i}$ and analyze its drift. Important: how does a single frequency evolve?

- Consider the two offspring $x$ and $y$ and look into bit $i$.

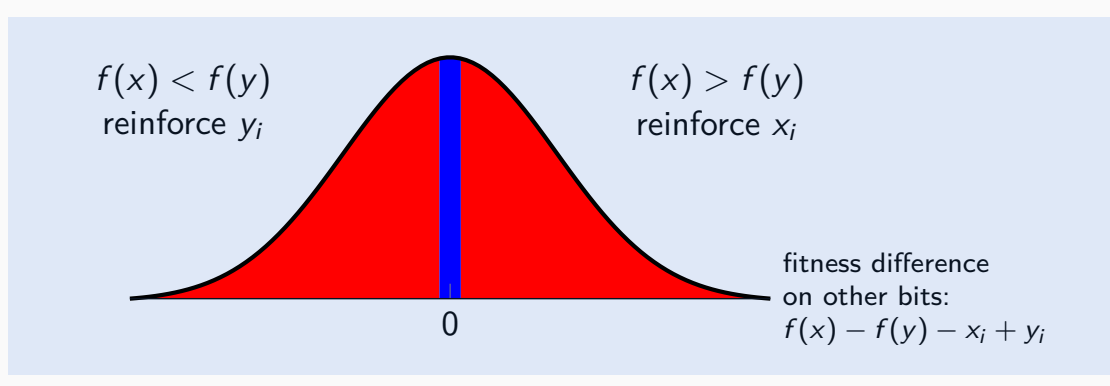

Red area: bit $i$ is irrelevant in this step

$\Rightarrow$ genetic drift moves $p_{t, i}$ in a random direction $\pm 1 / K$ (rw-step)

Blue area: bit $i$ decides the outcome of $f(x)$ vs. $f(y)$

$\Rightarrow$ increase $p_{t, i}$ (b-step, learning that $1 \mathrm{~s}$ are better than $0 \mathrm{~s}$ )

Probability of A Biased SteP at Bit $i$

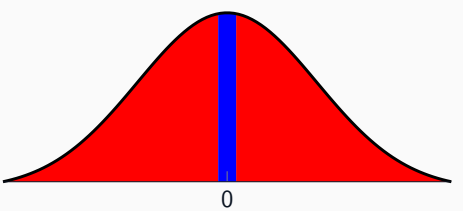

$\operatorname{Prob}($ b-step $) \sim 1 / \sqrt{n}$

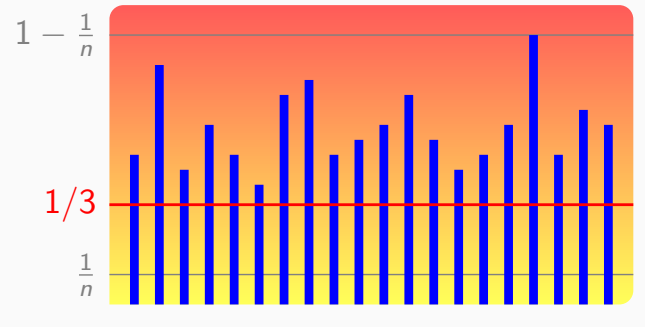

- Biased step occurs with probability at least $\Omega(1 / \sqrt{n})$. If offspring differ in the bit (prob. $2 p_{i}\left(1-p_{i}\right)$ ) then raised by $1 / K$.

- Otherwise, frequency is expected to stay put.

- Altogether:

$$
p_{t+1, i}=p_{t, i}+\Omega\left(p_{t, i}\left(1-p_{t, i}\right) / \sqrt{n}\right)
$$

Note similarity to $p_{t+1, i} \approx p_{t, i}+\frac{1}{\sqrt{n}} \sqrt{p_{t, i}\left(1-p_{t, i}\right)}$ by Mühlenbein and Mahnig (1999) of EDAs I OneMax

Probability of a Biased Step at Bit $i$

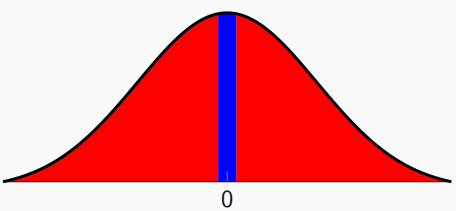

$\operatorname{Prob}($ b-step $) \sim 1 / \sqrt{n}$

- Biased step occurs with probability at least $\Omega(1 / \sqrt{n})$. If offspring differ in the bit (prob. $\left.2 p_{i}\left(1-p_{i}\right)\right)$ then raised by $1 / K$.

- Otherwise, frequency is expected to stay put.

- Altogether:

$$
p_{t+1, i}=p_{t, i}+\Omega\left(p_{t, i}\left(1-p_{t, i}\right) / \sqrt{n}\right)
$$

Note similarity to $p_{t+1, i} \approx p_{t, i}+\frac{1}{\sqrt{n}} \sqrt{p_{t, i}\left(1-p_{t, i}\right)}$ by Mühlenbein and Mahnig (1999) 
Now look into $K<\sqrt{n} \log n$ :

- Lower bound $\Omega(n)$ until 2015.

- Improved to $\Omega(n \log n)$ (Sudholt and W., 2016).

- Heavy genetic drift occurs.

\section{DEMO.}

Whether optimum can be found at all, depends very much on the borders on the frequencies

- If no borders, with high probability frequencies locked to $0 \Rightarrow$ infinite runtime.

- If borders $\{1 / n, 1-1 / n\}$ used, optimum can still be found in polynomial time! No proof for CGA, but for UMDA (Lehre and Nguyen, 2017; W., 2017)

$\Rightarrow$ Borders may make the algorithm efficient despite genetic drift.

Medium and Large $K$ : Overview

runtime

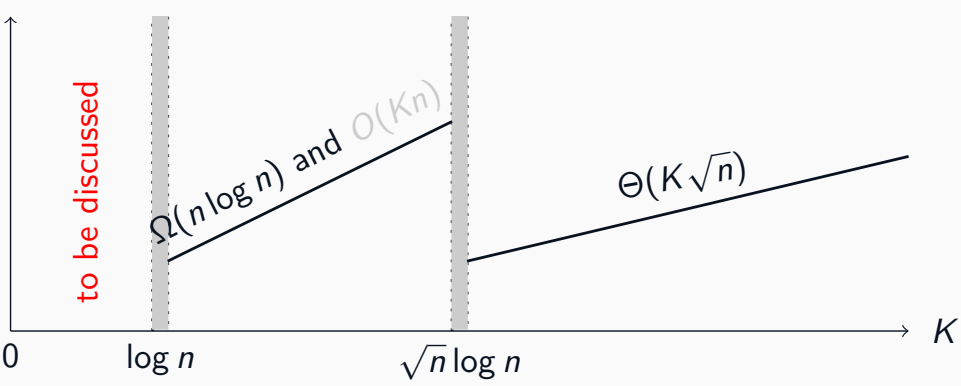

- Phase transition between smooth behavior and strong genetic drift at $K \sim \sqrt{n} \log n$

- If no borders, algorithm fails at $K=o(\sqrt{n} \log n)$

- With borders, efficient behavior as long as $K=\omega(\log n)$

Upper bound $O(K n)$ only conjectured here.

\section{Coupon collector}

You have to collect $n$ different coupons. In each round, you are given one coupon chosen uniformly at random with replacement. In expectation, it takes $\Omega(n \log n)$ rounds to collect all of them.

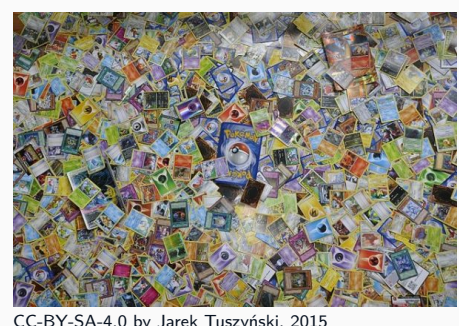

Here the coupons are the frequencies at the lower border. Each of them has probability $1 / n$ of being raised.

If many frequencies move to the lower border before optimum is found, we cannot be faster than $n \log n$.

\section{What happens if $K=o(\log n)$ ?}

Known for 2-MMAS ${ }_{\text {ib }}$ (Neumann et al., 2010), similar to cGA: landslides of frequencies occur.

\section{DEMO.}

Frequency that have attained their maximum $1-1 / n$ are nevertheless likely to drop down to minimum.

Very unstable behavior, exponential optimization time. 
Runtime of cGA on Onemax: Complete

\section{PICTURE}

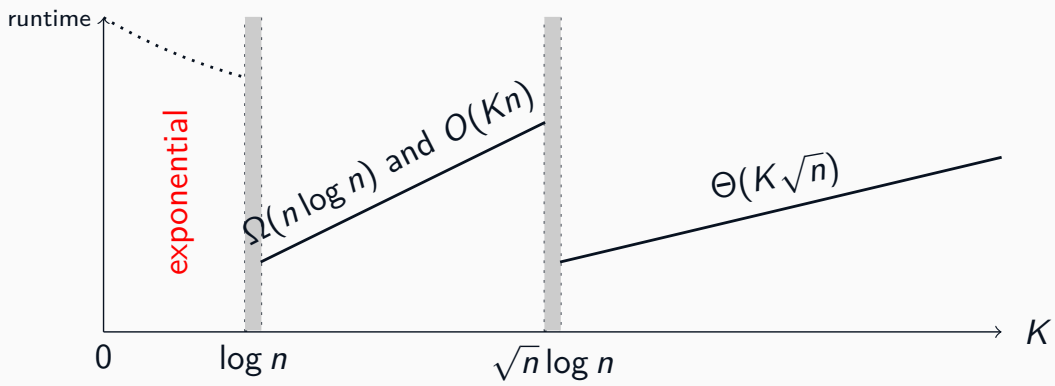

RESUlTS FOR UMDA: UPPER BOUNDS

First runtime result (Dang and Lehre, 2015): expected runtime of UMDA on ONEMAX is $O(n \lambda \log \lambda)$ for $\lambda>13 \mathrm{e} \mu$ and $\lambda=\Omega(\log n)$. Bound is $O(n \log n \log \log n)$ for best possible parameter setting.

Bound independently improved to $O(n \log n)$ by Lehre and Nguyen (2017) and W. (2017)

\section{Theorem (Expected runtime of UMDA on ONEMAX)}

1. For constant $a>0$ and constant $c \in(0,1)$, assume a $\ln n \leq \mu \leq \sqrt{n(1-c)}, \lambda \geq(13 \mathrm{e}) \mu /(1-c) \Rightarrow$ runtime $O(\lambda n)$.

2. Assume $\lambda=(1+\beta) \mu$ for constant $\beta>0, \mu \geq c \log n$ for large constant $c>0$, as well as $\mu=o(n) \Rightarrow$ runtime $O(\lambda n)$.

3. Assume $\lambda=(1+\beta) \mu$ for constant $\beta>0, \mu \geq c \sqrt{n} \log n$ for large constant $c>0 \Rightarrow$ runtime $O(\lambda \sqrt{n})$
ANALYSIS OF UMDA

How does UMDA perform on ONEMAX?

Surprisingly, in terms of runtimes (no. of iterations $\cdot \lambda$ ), not very differently from cGA

Obstacles in analysis:

- frequencies can change drastically, even from minimum to maximum in one generation,

- two parameters: $\mu$ and $\lambda$.

The first runtime analysis of UMDA on ONEMAX stems from 2015!
We also obtain similar bounds as with cGA:

Theorem (Krejca and W., 2017)

Let $\lambda=(1+\beta) \mu$ for some constant $\beta>0$ and $\lambda=n^{O(1)}$. Then the expected optimization time of UMDA on ONEMAX is $\Omega(\lambda \sqrt{n}+n \log n)$.

Proof idea again: at a very high level, we estimate

1. progress (drift) of sum of frequencies per iteration

2. time for genetic drift to move frequencies to wrong border both for upper and lower bounds. Especially 2. is challenging. 
Overview of Runtime Bound For UMDA on ONEMAX

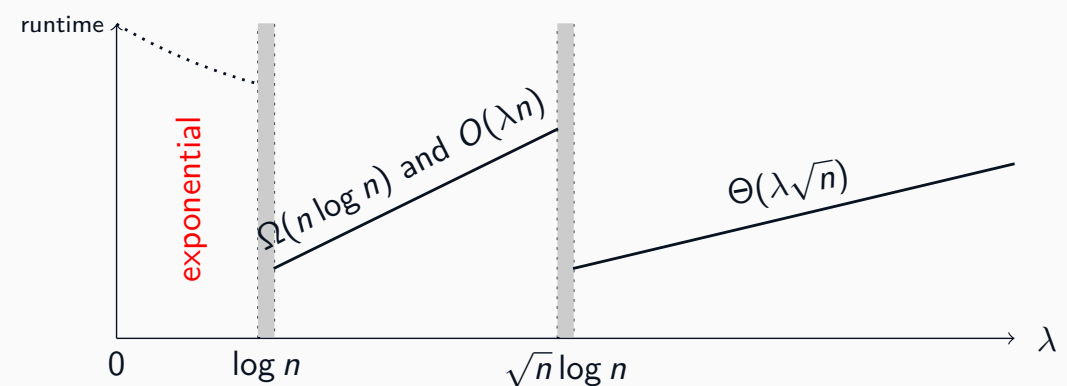

- Exponential time below $\log n$ strongly conjectured.

- Remaining bounds proved.

- If no borders on frequencies, runtime infinite below $\sqrt{n} \log n$
What Happens in the Medium Regime

If $\lambda=\omega(\log n)$ and $\lambda=o(\sqrt{n})$

$\Rightarrow$ expected runtime $O(\lambda n)$ and $\Omega(n \log n)$.

Take, e. g., $\lambda=n^{1 / 3} \Rightarrow$ upper bound $O\left(n^{4 / 3}\right)$, lower bound $\Omega(n \log n)$ Again upper bound $O(n \log n)$ for $\lambda=c \sqrt{n} \log n$. Where is the truth? Experiments:
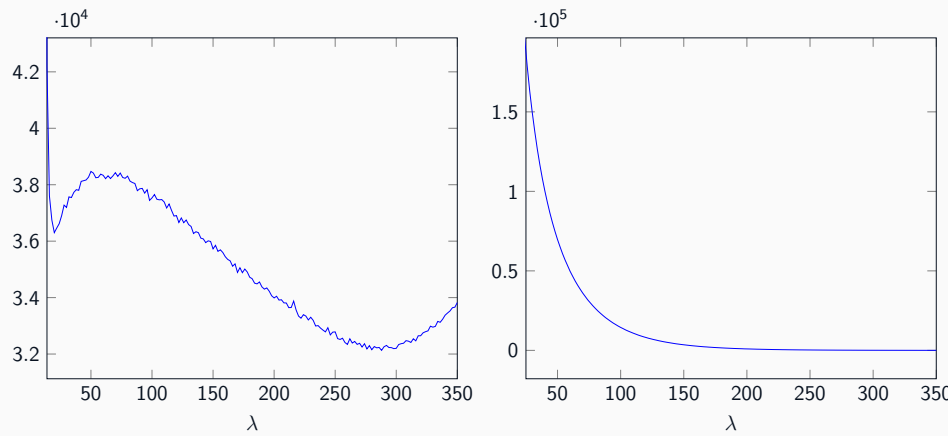

Left: average runtime; right: no. times frequency hits minimum In fact a multimodal behavior: is $O(\lambda n)$ also $\Omega(\lambda n)$ in medium regime?

New Result: Runtime of CGA is Multimodal

OnEMAX: Summary

- Simple univariate EDAs like UMDA and cGA (and 2-MMAS ${ }_{i b}$ ) have similar runtime behavior on ONEMAX.

- Very sensitive to settings of parameters.

- Two phase transitions.

- Multimodal behavior.

- If no borders are used, model must have model of preciseness $\Omega(\sqrt{n} \log n)$ to prevent genetic drift. 


\section{CONTENTS}

\section{Introduction \\ Preliminaries \\ OneMax \\ LeadingOnes \\ Binary Value \\ Noise \\ Jump \\ Stable EDAs \\ End}

\section{EDAS ON LEADINGONES}

$\operatorname{LEADINGONES}(x)=\sum_{i=1}^{n} \prod_{j=1}^{i} x_{j}$ has not been considered much in the theory of EDAs (except for UMDA)

Possible reason: behavior is more obvious than on ONEMAX and not very different from classical EAs.

Typical: frequencies are optimized from left to right. (DEMO)

$$
11110 * * * * * * * * * * * * * * *
$$

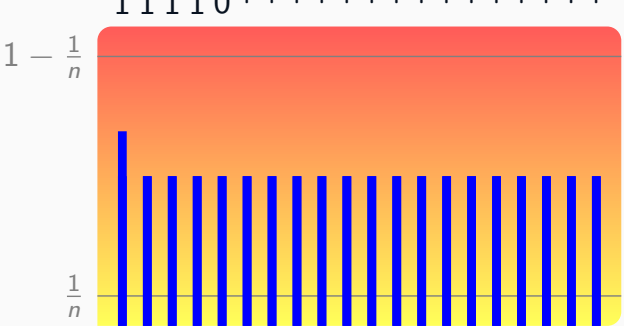

If best-so-far solution has $i$ leading ones, then last $n-i-1$ frequencies are drifting randomly.

EDAS ON LEADINGONES

LEADINGONES $(x)=\sum_{i=1}^{n} \prod_{j=1}^{i} x_{j}$ has not been considered much in the theory of EDAs (except for UMDA)

Possible reason: behavior is more obvious than on ONEMAX and not very different from classical EAs.

Typical: frequencies are optimized from left to right. (DEMO)

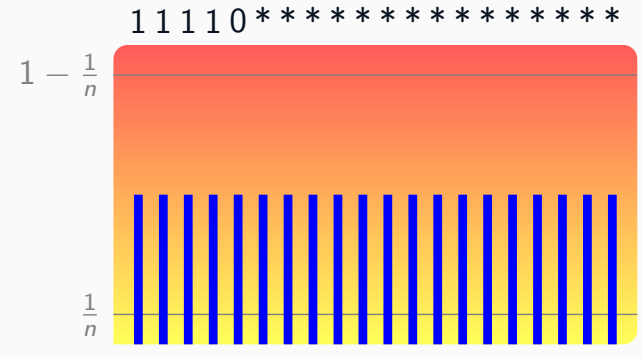

If best-so-far solution has $i$ leading ones, then last $n-i-1$ frequencies are drifting randomly.

EDAS ON LEADINGONES

LEADINGONES $(x)=\sum_{i=1}^{n} \prod_{j=1}^{i} x_{j}$ has not been considered much in the theory of EDAs (except for UMDA).

Possible reason: behavior is more obvious than on ONEMAX and not very different from classical EAs.

Typical: frequencies are optimized from left to right. (DEMO)

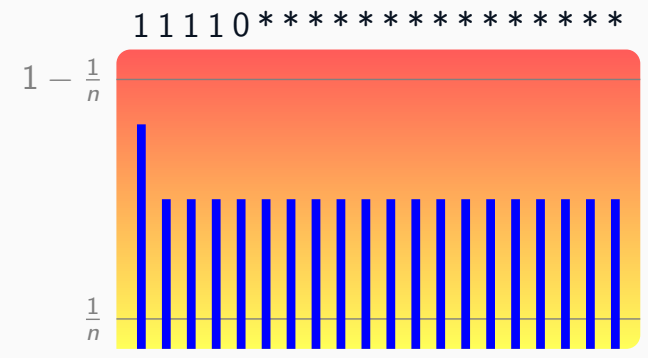

If best-so-far solution has $i$ leading ones, then last $n-i-1$ frequencies are drifting randomly. 
EDAS ON LEADINGONES

LEADINGOnES $(x)=\sum_{i=1}^{n} \prod_{j=1}^{i} x_{j}$ has not been considered much in the theory of EDAs (except for UMDA)

Possible reason: behavior is more obvious than on ONEMAX and not very different from classical EAs.

Typical: frequencies are optimized from left to right. (DEMO)

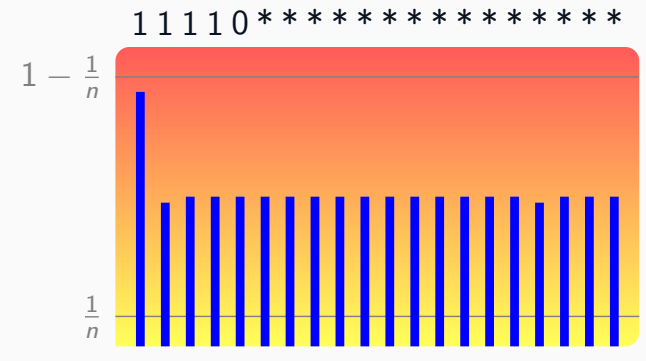

If best-so-far solution has $i$ leading ones, then last $n-i-1$ frequencies are drifting randomly.
EDAS ON LEADINGONES

LEADINGONES $(x)=\sum_{i=1}^{n} \prod_{j=1}^{i} x_{j}$ has not been considered much in the theory of EDAs (except for UMDA).

Possible reason: behavior is more obvious than on ONEMAX and not very different from classical EAs.

Typical: frequencies are optimized from left to right. (DEMO)

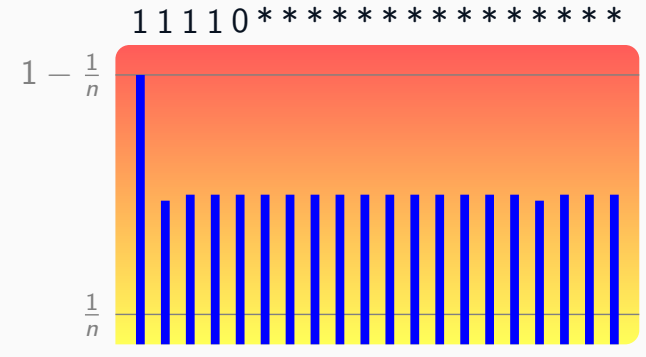

If best-so-far solution has $i$ leading ones, then last $n-i-1$ frequencies are drifting randomly.

\section{EDAS ON LEADINGONES}

$\operatorname{LEADINGONES}(x)=\sum_{i=1}^{n} \prod_{j=1}^{i} x_{j}$ has not been considered much in the theory of EDAs (except for UMDA).

Possible reason: behavior is more obvious than on ONEMAX and not very different from classical EAs.

Typical: frequencies are optimized from left to right. (DEMO)

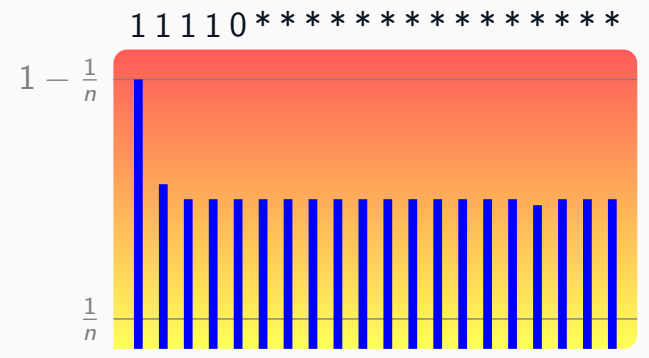

If best-so-far solution has $i$ leading ones, then last $n-i-1$ frequencies are drifting randomly.

EDAS ON LEADINGONES

$\operatorname{LEADING\operatorname {OnES}}(x)=\sum_{i=1}^{n} \prod_{j=1}^{i} x_{j}$ has not been considered much in the theory of EDAs (except for UMDA).

Possible reason: behavior is more obvious than on ONEMAX and not very different from classical EAs.

Typical: frequencies are optimized from left to right. (DEMO)

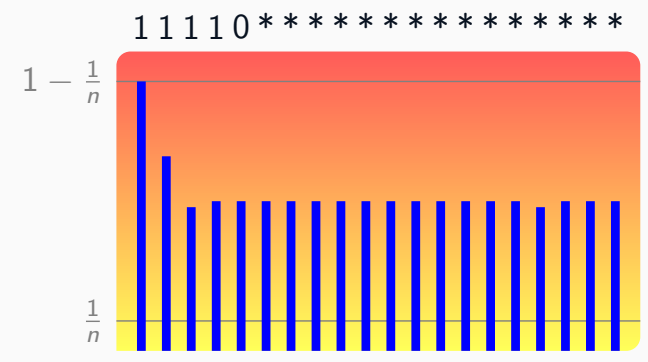

If best-so-far solution has $i$ leading ones, then last $n-i-1$ frequencies are drifting randomly. 
EDAS ON LEADINGONES

LEADINGOnES $(x)=\sum_{i=1}^{n} \prod_{j=1}^{i} x_{j}$ has not been considered much in the theory of EDAs (except for UMDA)

Possible reason: behavior is more obvious than on ONEMAX and not very different from classical EAs.

Typical: frequencies are optimized from left to right. (DEMO)

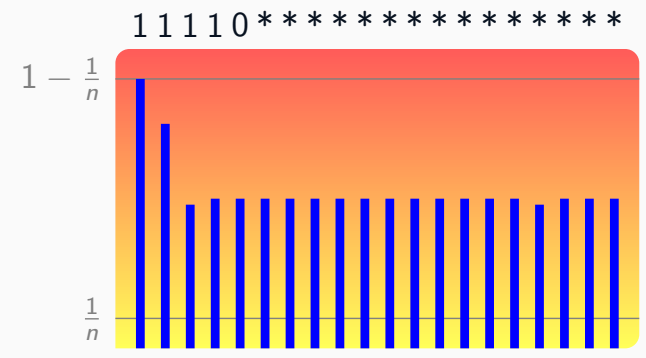

If best-so-far solution has $i$ leading ones, then last $n-i-1$ frequencies are drifting randomly.
EDAS ON LEADINGONES

LEADINGONES $(x)=\sum_{i=1}^{n} \prod_{j=1}^{i} x_{j}$ has not been considered much in the theory of EDAs (except for UMDA).

Possible reason: behavior is more obvious than on ONEMAX and not very different from classical EAs.

Typical: frequencies are optimized from left to right. (DEMO)

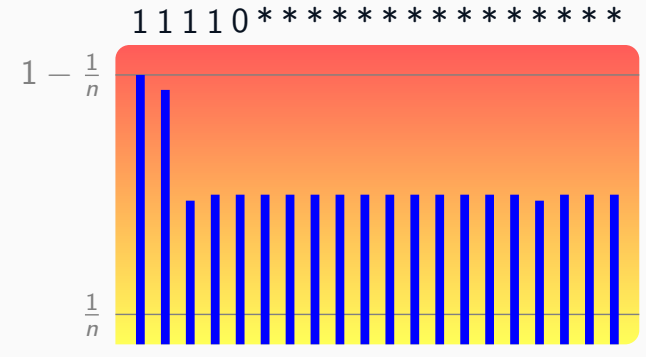

If best-so-far solution has $i$ leading ones, then last $n-i-1$ frequencies are drifting randomly.

\section{EDAS ON LEADINGONES}

$\operatorname{LEADINGONES}(x)=\sum_{i=1}^{n} \prod_{j=1}^{i} x_{j}$ has not been considered much in the theory of EDAs (except for UMDA).

Possible reason: behavior is more obvious than on ONEMAX and not very different from classical EAs.

Typical: frequencies are optimized from left to right. (DEMO)

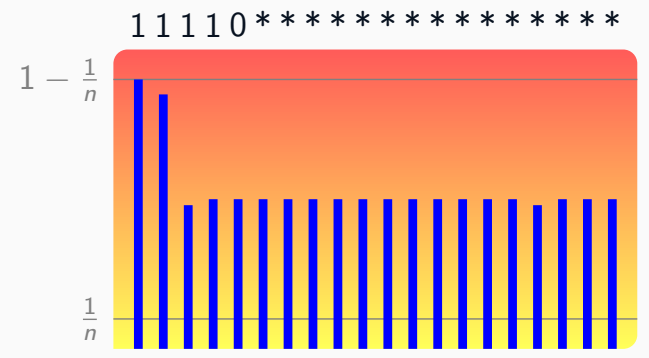

If best-so-far solution has $i$ leading ones, then last $n-i-1$ frequencies are drifting randomly.

EDAS ON LEADINGONES

$\operatorname{LEADING\operatorname {OnES}}(x)=\sum_{i=1}^{n} \prod_{j=1}^{i} x_{j}$ has not been considered much in the theory of EDAs (except for UMDA).

Possible reason: behavior is more obvious than on ONEMAX and not very different from classical EAs.

Typical: frequencies are optimized from left to right. (DEMO)

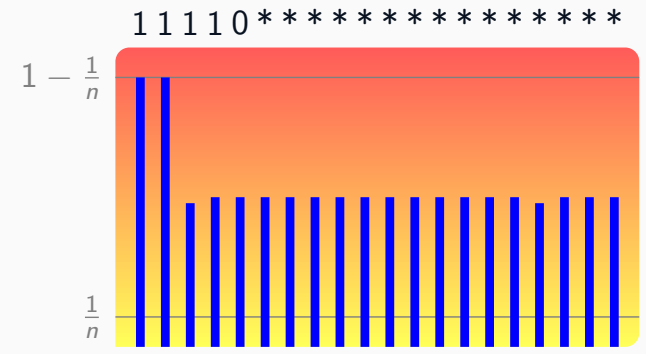

If best-so-far solution has $i$ leading ones, then last $n-i-1$ frequencies are drifting randomly. 
EDAS ON LEADINGONES

LEADINGOnES $(x)=\sum_{i=1}^{n} \prod_{j=1}^{i} x_{j}$ has not been considered much in the theory of EDAs (except for UMDA)

Possible reason: behavior is more obvious than on ONEMAX and not very different from classical EAs.

Typical: frequencies are optimized from left to right. (DEMO)

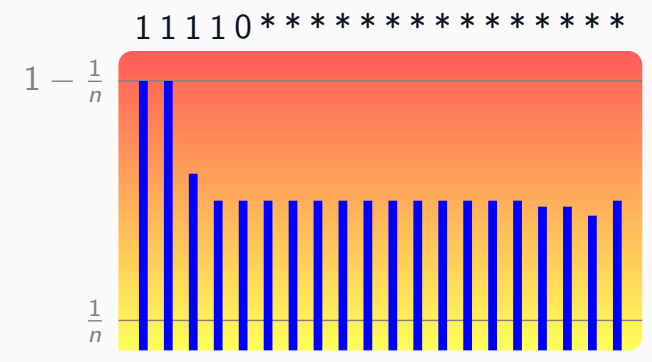

If best-so-far solution has $i$ leading ones, then last $n-i-1$ frequencies are drifting randomly.
EDAS ON LEADINGONES

LEADINGONES $(x)=\sum_{i=1}^{n} \prod_{j=1}^{i} x_{j}$ has not been considered much in the theory of EDAs (except for UMDA).

Possible reason: behavior is more obvious than on ONEMAX and not very different from classical EAs.

Typical: frequencies are optimized from left to right. (DEMO)

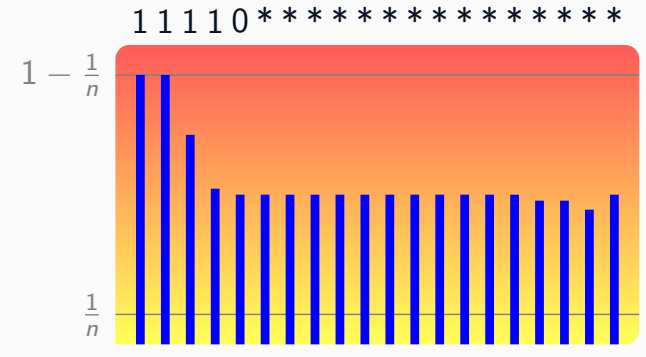

If best-so-far solution has $i$ leading ones, then last $n-i-1$ frequencies are drifting randomly.

\section{EDAS ON LEADINGONES}

$\operatorname{LEADINGONES}(x)=\sum_{i=1}^{n} \prod_{j=1}^{i} x_{j}$ has not been considered much in the theory of EDAs (except for UMDA).

Possible reason: behavior is more obvious than on ONEMAX and not very different from classical EAs.

Typical: frequencies are optimized from left to right. (DEMO)

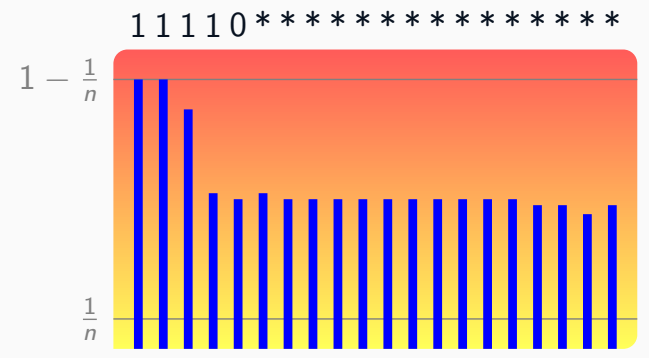

If best-so-far solution has $i$ leading ones, then last $n-i-1$ frequencies are drifting randomly.

EDAS ON LEADINGONES

$\operatorname{LEADING\operatorname {OnES}}(x)=\sum_{i=1}^{n} \prod_{j=1}^{i} x_{j}$ has not been considered much in the theory of EDAs (except for UMDA).

Possible reason: behavior is more obvious than on ONEMAX and not very different from classical EAs.

Typical: frequencies are optimized from left to right. (DEMO)

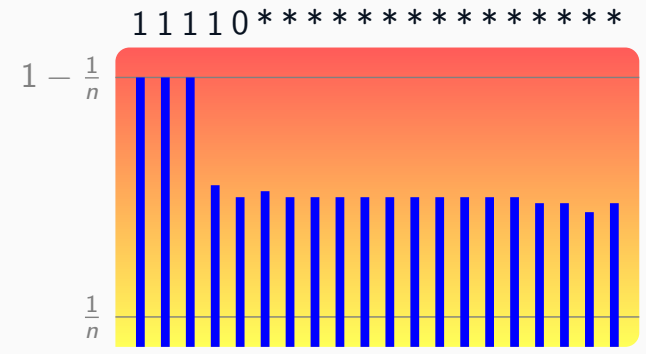

If best-so-far solution has $i$ leading ones, then last $n-i-1$ frequencies are drifting randomly. 
EDAS ON LEADINGONES

LEADINGOnES $(x)=\sum_{i=1}^{n} \prod_{j=1}^{i} x_{j}$ has not been considered much in the theory of EDAs (except for UMDA)

Possible reason: behavior is more obvious than on ONEMAX and not very different from classical EAs.

Typical: frequencies are optimized from left to right. (DEMO)

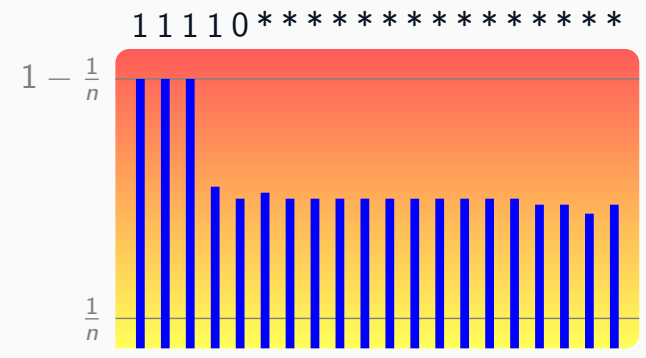

If best-so-far solution has $i$ leading ones, then last $n-i-1$ frequencies are drifting randomly.
EDAS ON LEADINGONES

LEADINGONES $(x)=\sum_{i=1}^{n} \prod_{j=1}^{i} x_{j}$ has not been considered much in the theory of EDAs (except for UMDA).

Possible reason: behavior is more obvious than on ONEMAX and not very different from classical EAs.

Typical: frequencies are optimized from left to right. (DEMO)

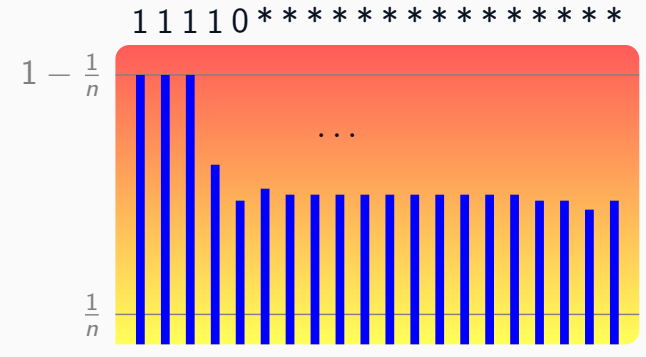

If best-so-far solution has $i$ leading ones, then last $n-i-1$ frequencies are drifting randomly.

\section{EDAS ON LEADINGONES}

$\operatorname{LEADINGONES}(x)=\sum_{i=1}^{n} \prod_{j=1}^{i} x_{j}$ has not been considered much in the theory of EDAs (except for UMDA)

Possible reason: behavior is more obvious than on ONEMAX and not very different from classical EAs.

Typical: frequencies are optimized from left to right. (DEMO)

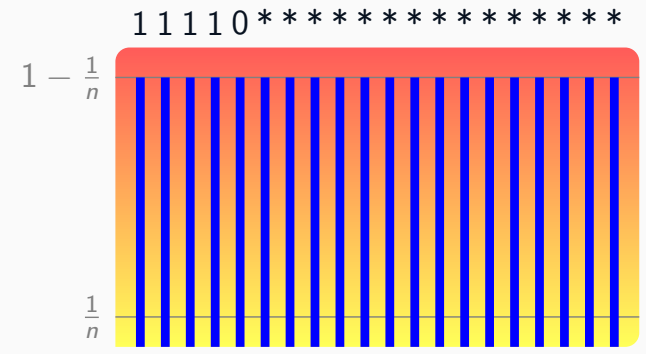

If best-so-far solution has $i$ leading ones, then last $n-i-1$ frequencies are drifting randomly.

Early Results for LeadingOnes

UMDA without borders (Chen et al., 2007, 2009b, 2010):

Using $\lambda=\Omega\left(n^{2+\varepsilon}\right)$, UMDA (without borders) optimizes LEADINGONES in time $O(\lambda n)$ w. h. p. $\Rightarrow$ runtime $O\left(n^{3+\varepsilon}\right)$ for optimal $\lambda$.

For comparison: (1+1) EA expected runtime $\Theta\left(n^{2}\right)$.

Approach in the analysis again:

1. Determine total time for frequency vector to converge to optimality.

2. Determine lower bound on preciseness of model to prevent genetic drift before convergence.

Large $\lambda$ used to prevent genetic drift also for the last optimized bit (possibly too large). 
Use the borders.

Theorem (Dang and Lehre, 2015)

If $\lambda \geq c \ln n$ then expected runtime of UMDA with borders on

LEADINGONES is $O\left(n \lambda \log \lambda+n^{2}\right)$.

Holds also for PBIL (generalized UMDA) if learning rate not too small

(Lehre and Nguyen, 2018).

EDAs BeAt EAs on Substring

Theorem (simplified from Chen et al., 2009a)

$(1+1) E A$ needs with overwhelming probability time $2^{c n}$ to optimize SUBSTRING. UMDA with $\lambda=\Omega\left(n^{2+\varepsilon}\right), \mu=\lambda / 2$ optimizes SUBSTRING in polynomial time w. o. p.

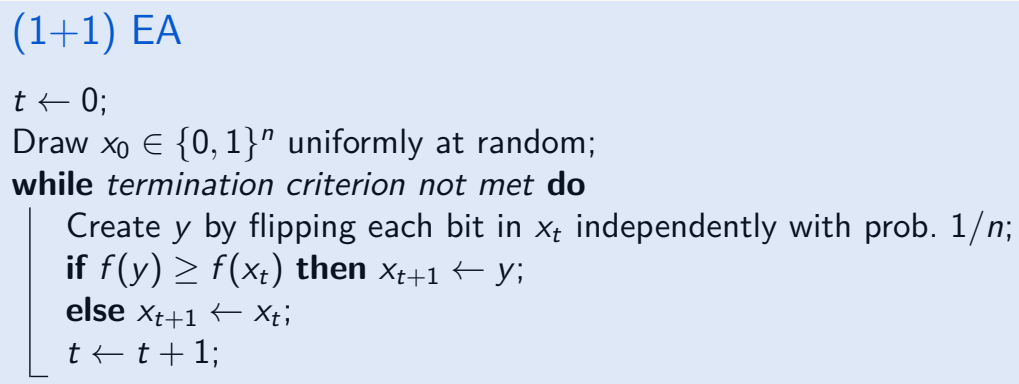

Overall analysis was similar to ONEMAX. No new insights through the study of LEADINGONES?

Consider following example from Chen et al. (2009a).

$$
\operatorname{SubStRing}(x)= \begin{cases}2 n & \text { if } x=(1, \ldots, 1) \\ \max _{i=1}^{n} i \cdot \prod_{j=\max \{i-n / 4,1\}}^{i} x_{j} & \text { otherwise }\end{cases}
$$

Substring equals LEADingOnes if no block of at least $n / 4$ consecutive ones. E. g.: $11110 * * * * * * * * * \mapsto 4$

Otherwise, it describes the starting position of the rightmost block of $n / 4$ ones - except if everything is one. E. g.: $010101011 \ldots 111 \mapsto 3 n / 4$

Theorem (simplified from Chen et al., 2009a)

$(1+1) E A$ needs with overwhelming probability time $2^{\text {cn }}$ to optimize SUBSTRING. UMDA with $\lambda=\Omega\left(n^{2+\varepsilon}\right), \mu=\lambda / 2$ optimizes SUBSTRING in polynomial time w. o.p.

\section{Proof idea for $(1+1)$ EA}

Typically, $(1+1)$ EA starts out by gaining more and more leading ones.

$$
10 * * * * * * * * * * * * * * * * * * * * * * * * * *
$$

When $>n / 4$ leading ones, the first bit(s) no longer contributes to fitness. These bits slowly become random again. Optimum missed, NIAH 
Theorem (simplified from Chen et al., 2009a)

$(1+1) E A$ needs with overwhelming probability time $2^{\text {cn }}$ to optimize SUBSTRING. UMDA with $\lambda=\Omega\left(n^{2+\varepsilon}\right), \mu=\lambda / 2$ optimizes SUBSTRING in polynomial time w. o. p.

Proof idea for $(1+1)$ EA

Typically, $(1+1)$ EA starts out by gaining more and more leading ones.

\section{$110 * * * * * * * * * * * * * * * * * * * * * * * * *$}

When $>n / 4$ leading ones, the first bit(s) no longer contributes to fitness.

These bits slowly become random again. Optimum missed, NIAH

EDAs BeAt EAs on Substring

Theorem (simplified from Chen et al., 2009a)

$(1+1)$ EA needs with overwhelming probability time $2^{\text {cn }}$ to optimize SubSTRING. UMDA with $\lambda=\Omega\left(n^{2+\varepsilon}\right), \mu=\lambda / 2$ optimizes SUBSTRING in polynomial time w. o.p.

\section{Proof idea for $(1+1)$ EA}

Typically, $(1+1)$ EA starts out by gaining more and more leading ones.

\section{$111110 * * * * * * * * * * * * * * * * * * * * * *$}

When $>n / 4$ leading ones, the first bit(s) no longer contributes to fitness.

These bits slowly become random again. Optimum missed, NIAH

Theorem (simplified from Chen et al., 2009a)

$(1+1) E A$ needs with overwhelming probability time $2^{c n}$ to optimize SUBSTRING. UMDA with $\lambda=\Omega\left(n^{2+\varepsilon}\right), \mu=\lambda / 2$ optimizes SUBSTRING in polynomial time w. o.p.

Proof idea for $(1+1)$ EA

Typically, $(1+1)$ EA starts out by gaining more and more leading ones.

\section{$1110 * * * * * * * * * * * * * * * * * * * * * * * *$}

When $>n / 4$ leading ones, the first bit(s) no longer contributes to fitness.

These bits slowly become random again. Optimum missed, NIAH

EDAs Beat EAs on Substring

Theorem (simplified from Chen et al., 2009a)

$(1+1)$ EA needs with overwhelming probability time $2^{\text {cn }}$ to optimize SUBSTRING. UMDA with $\lambda=\Omega\left(n^{2+\varepsilon}\right), \mu=\lambda / 2$ optimizes SUBSTRING in polynomial time w. o.p.

Proof idea for $(1+1)$ EA

Typically, $(1+1)$ EA starts out by gaining more and more leading ones.

\section{$11111111111110 * * * * * * * * * * * * *$}

When $>n / 4$ leading ones, the first bit(s) no longer contributes to fitness.

These bits slowly become random again. Optimum missed, NIAH 
Theorem (simplified from Chen et al., 2009a)

$(1+1) E A$ needs with overwhelming probability time $2^{\text {cn }}$ to optimize SUBSTRING. UMDA with $\lambda=\Omega\left(n^{2+\varepsilon}\right), \mu=\lambda / 2$ optimizes SUBSTRING in polynomial time w. o. p.

Proof idea for $(1+1)$ EA

Typically, $(1+1)$ EA starts out by gaining more and more leading ones.

\section{$11111111111111110 * * * * * * * * * *$}

When $>n / 4$ leading ones, the first bit(s) no longer contributes to fitness.

These bits slowly become random again. Optimum missed, NIAH

EDAs Beat EAs on Substring

Theorem (simplified from Chen et al., 2009a)

$(1+1)$ EA needs with overwhelming probability time $2^{c n}$ to optimize SUBSTRING. UMDA with $\lambda=\Omega\left(n^{2+\varepsilon}\right), \mu=\lambda / 2$ optimizes SUBSTRING in polynomial time w. o.p.

\section{Proof idea for $(1+1)$ EA}

Typically, $(1+1)$ EA starts out by gaining more and more leading ones.

\section{$1101111111111111110 * * * * * * * * *$}

When $>n / 4$ leading ones, the first bit(s) no longer contributes to fitness.

These bits slowly become random again. Optimum missed, NIAH
Theorem (simplified from Chen et al., 2009a)

$(1+1) E A$ needs with overwhelming probability time $2^{c n}$ to optimize SUBSTRING. UMDA with $\lambda=\Omega\left(n^{2+\varepsilon}\right), \mu=\lambda / 2$ optimizes SUBSTRING in polynomial time w. o.p.

Proof idea for $(1+1)$ EA

Typically, $(1+1)$ EA starts out by gaining more and more leading ones.

\section{$1101111111111111110 * * * * * * * * *$}

When $>n / 4$ leading ones, the first bit(s) no longer contributes to fitness.

These bits slowly become random again. Optimum missed, NIAH

EDAs Beat EAs on Substring

Theorem (simplified from Chen et al., 2009a)

$(1+1) E A$ needs with overwhelming probability time $2^{\text {cn }}$ to optimize SUBSTRING. UMDA with $\lambda=\Omega\left(n^{2+\varepsilon}\right), \mu=\lambda / 2$ optimizes SUBSTRING in polynomial time w. o.p.

Proof idea for $(1+1)$ EA

Typically, $(1+1)$ EA starts out by gaining more and more leading ones. $1100111111111111110 * * * * * * * *$

When $>n / 4$ leading ones, the first bit(s) no longer contributes to fitness.

These bits slowly become random again. Optimum missed, NIAH 
Theorem (simplified from Chen et al., 2009a)

$(1+1) E A$ needs with overwhelming probability time $2^{\text {cn }}$ to optimize SUBSTRING. UMDA with $\lambda=\Omega\left(n^{2+\varepsilon}\right), \mu=\lambda / 2$ optimizes SUBSTRING in polynomial time w. o. p.

Proof idea for $(1+1)$ EA

Typically, $(1+1)$ EA starts out by gaining more and more leading ones. $110010111111111111110 * * * * * *$

When $>n / 4$ leading ones, the first bit(s) no longer contributes to fitness. These bits slowly become random again. Optimum missed, NIAH
Theorem (simplified from Chen et al., 2009a)

$(1+1) E A$ needs with overwhelming probability time $2^{\text {cn }}$ to optimize SUBSTRING. UMDA with $\lambda=\Omega\left(n^{2+\varepsilon}\right), \mu=\lambda / 2$ optimizes SUBSTRING in polynomial time w. o.p.

Proof idea for $(1+1)$ EA

Typically, $(1+1)$ EA starts out by gaining more and more leading ones. 110010101001111111111111111

When $>n / 4$ leading ones, the first bit(s) no longer contributes to fitness. These bits slowly become random again. Optimum missed, NIAH

\section{Proof idea for UMDA}

Also UMDA starts out by gaining more and more leading ones. This is reflected in the frequencies.

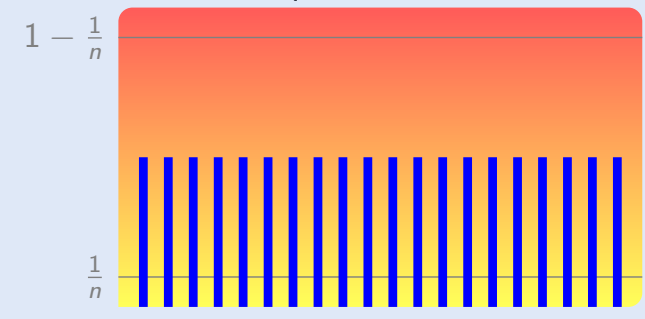

10101110110111011010

Even after the first bits no longer contribute to fitness, their frequencies are expected to remain the same. All-ones string can be sampled.

Need large enough $\lambda$ to prevent genetic drift.

\section{Proof idea for UMDA}

Also UMDA starts out by gaining more and more leading ones. This is reflected in the frequencies.

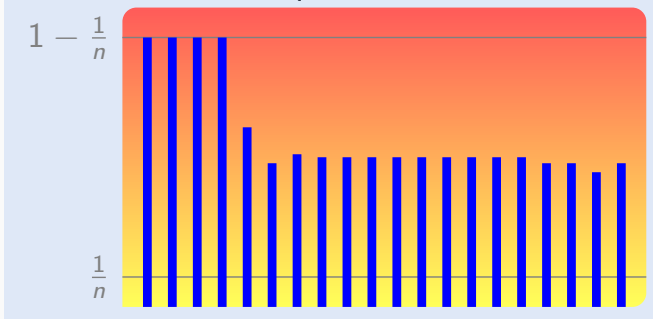

$11110 * * * * * * * * * * * * * * *$

Even after the first bits no longer contribute to fitness, their frequencies are expected to remain the same. All-ones string can be sampled.

Need large enough $\lambda$ to prevent genetic drift. 


\section{Proof idea for UMDA}

Also UMDA starts out by gaining more and more leading ones. This is reflected in the frequencies.

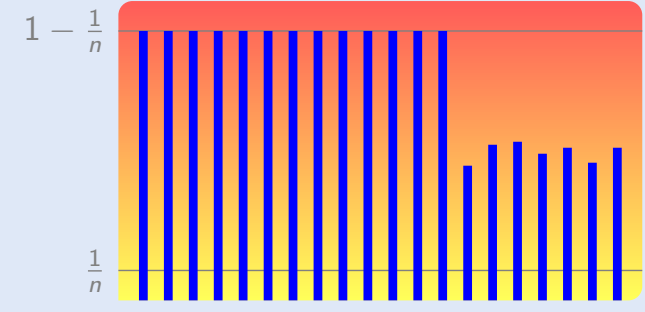

$1111111111111110 * * * *$

Even after the first bits no longer contribute to fitness, their frequencies are expected to remain the same. All-ones string can be sampled.

Need large enough $\lambda$ to prevent genetic drift.
Proof idea for UMDA

Also UMDA starts out by gaining more and more leading ones. This is reflected in the frequencies.

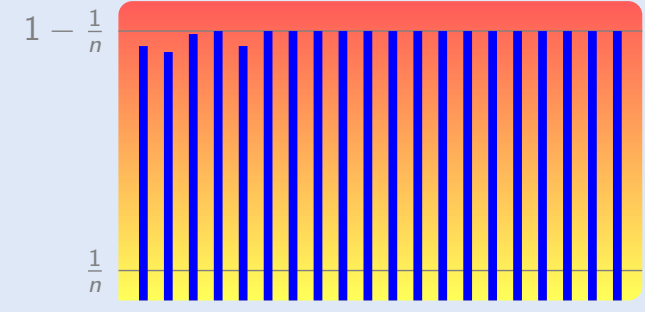

1111111111111111111

Even after the first bits no longer contribute to fitness, their frequencies are expected to remain the same. All-ones string can be sampled.

Need large enough $\lambda$ to prevent genetic drift.

Typical behavior of EDAs:

- On OneMax they optimize all bits roughly at the same time.

- On LeadingOnes they optimize bits from left to the right

- Runtime $\Theta(n \log n)$ vs. $\Theta\left(n^{2}\right)$ for opt. parameters settings.

$>$

However, there are other EDAs that do not behave like this (see later)

of EDAs ） BinaryValue

Contents

Introduction

Preliminaries

OneMax

LeadingOnes

BinaryValue

Noise

Jump

Stable EDAs

End 
The Final EXAmple Function: BinVal

Recall

$$
\operatorname{BinVAL}\left(x_{1}, \ldots, x_{n}\right):=\sum_{i=1}^{n} 2^{n-i} x_{i}
$$

being somewhere between LEADINGOnes and OneMax. A bit outweighs all less significant bits together, but every bit contributes to fitness.

Often, a runtime analysis of LEADINGONES also gives a runtime bound for BinVAL (e. g., for UMDA/PBIL, Lehre and Nguyen, 2018).

\section{Theorem (Droste, 2006)}

The runtime of $c G A$ on BINVAL is $O(K n)$ for $K=\Omega\left(n^{1+\varepsilon}\right)$ w. o. p. It is $\Omega(K n)$ w. o. p.

Note: upper bound $O\left(n^{2+\varepsilon}\right)$. Lower bound does not restrict $K$.
Not all Linear Functions Are Equally

DIFFICULT FOR EDAS

Known for (1+1) EA (W., 2013): all linear functions optimized in expected time $(1 \pm o(1))$ en $\ln n$.

$\Rightarrow$ Runtimes on ONEMAX and BinVAL differ by a lower-order term.

Droste conjectured that BINVAL cannot be optimized in time $O(n \log n)$.

Theorem (W., 2018)

The runtime of cGA (without borders) on BINVAL is $\Omega\left(n^{2}\right)$ with prob. $\Omega(1)$. Choosing $K=o(n)$ leads to infinite runtime w. h. $p$.

Idea: if $K$ too small, genetic drift likely to occur at light bits before all heavy bits optimized.

\section{CONTENTS}

Introduction

Preliminaries

OneMax

LeadingOnes

Binary Value

Noise

Jump

Stable EDAs

End of EDAs I Nois

OPtimizATION UNDER UNCERTAINTY

Assume that an evaluation of the objective function is subject to random noise.

Example: ONEMAX with additive Gaussian noise

$f_{\text {noise }}(x)=\operatorname{OnEMAx}(x)+N(0, \sigma)$.

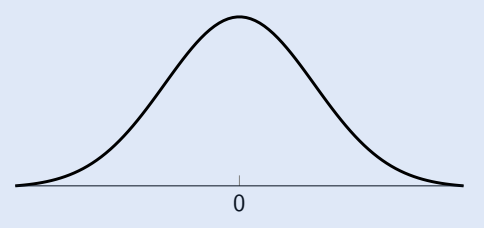

Typical measures to handle noise in evolutionary computation:

- large populations

- resampling

EDAs have a built-in noise handling mechanism. 
EDAs Beat Population-Based EAs in Noisy SETTINGS

Definition (Friedrich et al., 2017): an algorithm scales gracefully if its expected runtime depends polynomially on the noise strength.

For example: runtime is proportionally to $\sigma^{2}$.

\section{Theorem}

$(\mu+1)$ EA does not scale gracefully on ONEMAX with additive Gaussian noise. However, cGA does.
Proof IDEAS

- $(\mu+1)$ EA uses mutation, which drifts away from the optimum when there are many correct bits and noise disturbs fitness signal.

$$
\begin{aligned}
& x=11111111011111111111011111 \\
& \begin{array}{l}
\downarrow \\
y=11110111011111111111011111
\end{array} \quad \operatorname{Prob}(f(x)>f(y)) \approx \operatorname{Prob}(f(x)<f(y))
\end{aligned}
$$

- cGA does not use mutation and is balanced: in expectation, frequency vector and thus fitness does not decrease over time.

$1-\frac{1}{n}$

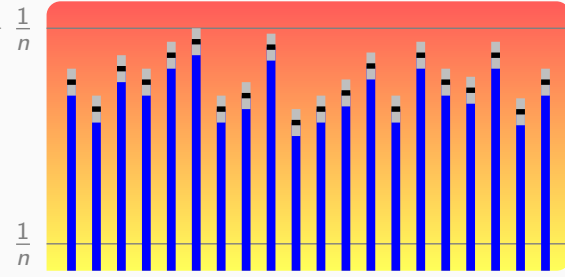

- cGA does not receive negative signal from noise, but signal (drift) towards increasing ONEMAX becomes smaller with increasing $\sigma$.

- Have to ensure that effect of genetic drift is smaller than the signal $\rightarrow$ choose big enough $K$.

Further Noisy SETtings

Also simple ACO algorithms can be considered EDAs and are superior to EAs in some noisy settings (also from combinatorial optimization):

- Sudholt and Thyssen (2012) for ACO for noisy shortest paths with a ground truth

- Doerr et al. (2012) where the noise is intrinsic

- Feldmann and Kötzing (2013) for ACO with fitness-proportional updates: leads to convergence to expected best solution

Many of the results can probably be transferred to more classical EDAs such as CGA and UMDA.

Contents
Introduction
Preliminaries
OneMax
LeadingOnes
Binary Value
Noise
Jump 


\title{
How EDAs Overcome GaPs
}

JUMP challenging for hillclimbers: $\Theta\left(n^{k}\right)$ to cross gap of size $k>1$.

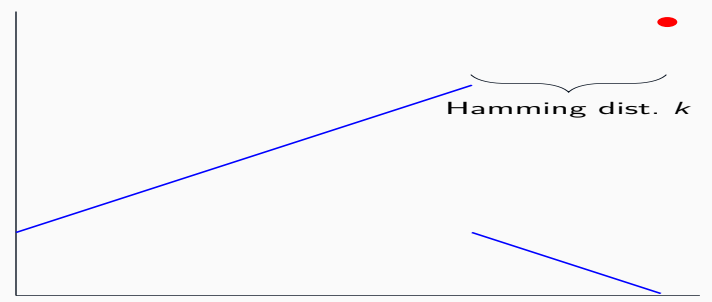

\section{CONTENTS}

\author{
Introduction \\ Preliminaries \\ OneMax \\ LeadingOnes \\ Binary Value \\ Noise \\ Jump
}

Stable EDAs

If $k \leq \frac{1}{20} \ln n$ and $\mu=\Omega(\sqrt{n} \log n)$ then $c G A$ optimizes Jump in $O(\mu \sqrt{n})$ iterations w. h. p. Time $O(n \log n)$ if $\mu=\Theta(\sqrt{n} \log n)$.

See talk at this GECCO.

\section{Balancedness, Stability and Genetic Drift}

Have seen: without fitness signal, stochastic model of EDAs is expected to be the same. Term: balanced (Friedrich et al., 2016a).

However, this is only the expected value. Genetic drift plays major role in classical EDAs.
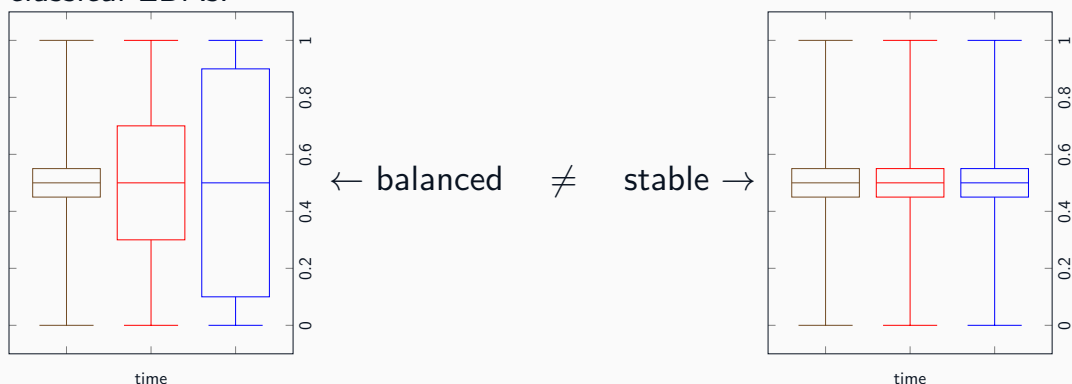

Term: EDA is stable if a frequency in absence of fitness signal stays close to its initial value.

cGA, UMDA, ... are not stable. Frequencies quickly converge to either maximum or minimum (each with probability $1 / 2$ ) due to genetic drift.

A New Way to Overcome Genetic Drift:

\section{SIGNIFICANCE-BASED EDAs}

Idea (Doerr and Krejca, 2018): Move frequency away from its initial value only when there is evidence that 0 or 1 is the better bit value.

sig-cGA: algorithmic ideas

- Framework like cGA.

- For each bit, history $H_{i} \in\{0,1\}^{*}$ of values in better individual

- Investigate last $m$ history bits. If a value significantly dominates, move frequency to corresponding border $(1 / n$ if 0 dominates, $1-1 / n$ if 1$)$.

- Otherwise, leave frequency at $1 / 2$.

- Example of significance: ||$\left|H_{i} \|_{1}-\frac{m}{2}\right| \geq C \sqrt{m \ln n}$

- Different values for $m$ are tested by the algorithm. 


\section{CONTEnTS}

\section{Theorem \\ The expected runtime of sig-cGA on both ONEMAX ond LEADINGONES is $O(n \log n)$ (and with high probability).}

No other evolutionary algorithm is known that simultaneously optimizes OneMaX and LEADINGONES in time $O(n \log n)$.

\section{Proof ideas}

- On OneMax, drift $\Omega\left(p_{i}\left(1-p_{i}\right) / \sqrt{n}\right)$ quickly identified as significant. Many ideas of analysis of plain cGA work.

- On LeadingOnes, frequencies are optimized from left to right. Bits that do not contribute to fitness yet: no signal, no significance of deviation, no genetic drift, stay at $1 / 2$

Significance-based EDAs are promising, theory-driven approach.

\section{Introduction \\ Preliminaries \\ OneMax \\ LeadingOnes \\ Binary Value \\ Noise \\ Jump \\ Stable EDAs}

End

\section{Summary AND CONCLUSIONS}

\section{Summary}

- Runtime analysis for simple univariate EDAs

- Identified similarities to and differences from simple EAs

- Genetic drift a major obstacle

- Sensitive to parameters (phase transitions)

- Robust to noise

- Significance-based EDAs as novel theory-driven approach

\section{Future work}

- Combinatorial problems

- Multivariate EDAs

- Classification of problems w. r.t. appropriateness for EAs/EDAs, ...

\section{Book chapter related to this tutorial}

Martin Krejca and Carsten Witt, Theory of Estimation-of-Distribution Algorithms, in: B. Doerr and F. Neumann (editors), Theory of Randomized Search Heuristics in Discrete Search Spaces, Springer, to appear https://arxiv.org/abs/1806.05392

REFERENCES I

$\mathrm{H}$. Asoh and H. Mühlenbein. On the mean convergence time of evolutionary algorithms without selection and mutation. In Proc. of PPSN '94, pages 88-97. Springer, 1994.

S. Baluja. Population-based incremental learning: A method for integrating genetic search based function optimization and competitive learning. Technical Report CMU-CS-94-163, Carnegie Mellon University, Pittsburgh, PA, 1994.

T. Chen, K. Tang, G. Chen, and X. Yao. On the analysis of average time complexity of estimation of distribution algorithms. In Proc. of CEC '07, pages 453-460. IEEE Press, 2007.

T. Chen, P. K. Lehre, K. Tang, and X. Yao. When is an estimation of distribution algorithm better than an evolutionary algorithm? In Proc. of CEC '09, pages 1470-1477. IEEE Press, 2009a.

T. Chen, K. Tang, G. Chen, and X. Yao. Rigorous time complexity analysis of univariate marginal distribution algorithm with margins. In Proc. of CEC '09, pages 2157-2164. IEEE Press, 2009b. 
REFERENCES II

T. Chen, K. Tang, G. Chen, and X. Yao. Analysis of computational time of simple estimation of distribution algorithms. IEEE Transactions on Evolutionary Computation, 14(1):1-22, 2010.

D. Corus, D.-C. Dang, A. V. Eremeev, and P. K. Lehre. Level-based analysis of genetic algorithms and other search processes. IEEE Transactions on Evolutionary Computation, 22(5):707-719, 2018.

D. Dang and P. K. Lehre. Simplified runtime analysis of estimation of distribution algorithms. In Proc. of GECCO '15, pages 513-518. ACM Press, 2015

J. S. De Bonet, C. L. Isbell, Jr, and P. A. Viola. MIMIC: Finding optima by estimating probability densities. In Proc. of NIPS '96, pages 424-430. MIT Press, 1997.

B. Doerr. A tight runtime analysis for the cGA on Jump functions - EDAs can cross fitness valleys at no extra cost. In Proc. of GECCO '19. ACM Press, 2019. To appear.
REFERENCES III

B. Doerr and M. S. Krejca. Significance-based estimation-of-distribution algorithms. In Proc. of GECCO 2018, pages 1483-1490, 2018.

B. Doerr, A. Hota, and T. Kötzing. Ants easily solve stochastic shortest path problems. In Proc. of GECCO '12, pages 17-24. ACM Press, 2012.

C. Doerr and J. Lengler. Onemax in black-box models with several restrictions. In Proc. of GECCO '15, pages 1431-1438. ACM Press, 2015.

S. Droste. A rigorous analysis of the compact genetic algorithm for linear functions. Natural Computing, 5(3):257-283, 2006.

M. Feldmann and T. Kötzing. Optimizing expected path lengths with ant colony optimization using fitness proportional update. In Proc. of FOGA '13, pages 65-74. ACM Press, 2013.

T. Friedrich, T. Kötzing, and M. S. Krejca. EDAs cannot be balanced and stable. In Proc. of GECCO '16, pages 1139-1146. ACM Press, 2016 a.

\section{REFERENCES IV}

T. Friedrich, T. Kötzing, M. S. Krejca, and A. M. Sutton. Robustness of ant colony optimization to noise. Evolutionary Computation, 24(2): 237-254, 2016b.

T. Friedrich, T. Kötzing, M. S. Krejca, and A. M. Sutton. The compact genetic algorithm is efficient under extreme gaussian noise. IEEE Transactions on Evolutionary Computation, 21(3):477-490, 2017.

Y. Gao and J. Culberson. Space complexity of estimation of distribution algorithms. Evolutionary Computation, 13(1):125-143, 2005.

C. González, J. Lozano, and P. Larrañaga. Analyzing the PBIL algorithm by means of discrete dynamical systems. Complex Systems, 12(4): 465-479, 2000.

G. R. Harik, F. G. Lobo, and K. Sastry. Linkage learning via probabilistic modeling in the extended compact genetic algorithm (ECGA). In Scalable Optimization via Probabilistic Modeling: From Algorithms to Applications Pelikan et al. (2006), pages 39-61.

REFERENCES V

V. Hasenöhrl and A. M. Sutton. On the runtime dynamics of the compact genetic algorithm on Jump functions. In Proc. of GECCO 18,, pages 967-974. ACM Press, 2018

M. Hauschild and M. Pelikan. An introduction and survey of estimation of distribution algorithms. Swarm and Evolutionary Computation, 1(3): 111-128, 2011

M. Höhfeld and G. Rudolph. Towards a theory of population-based incremental learning. In Proc. of CEC 97, pages 1-5. IEEE Press, 1997.

J. Kacprzyk and W. Pedrycz, editors. Springer Handbook of Computational Intelligence. Springer, 2015.

M. S. Krejca and C. W. Lower bounds on the run time of the univariate marginal distribution algorithm on OneMax. In Proc. of FOGA '17, pages 65-79. ACM Press, 2017.

P. Larrañaga and J. A. Lozano. Estimation of Distribution Algorithms: A New Tool for Evolutionary Computation, volume 2 of Genetic Algorithms and Evolutionary Computation. Springer, 2002. 


\section{REFERENCES VI}

P. K. Lehre and P. T. H. Nguyen. Improved runtime bounds for the univariate marginal distribution algorithm via anti-concentration. In Proc. of GECCO '17, pages 1383-1390. ACM Press, 2017.

P. K. Lehre and P. T. H. Nguyen. Level-based analysis of the population-based incremental learning algorithm. In Proc. of PPSN '18, volume 11102 of LNCS, pages 105-116. Springer, 2018.

P. K. Lehre and C. Witt. Black-box search by unbiased variation. In Proc. of GECCO '10, pages 1441-1448. ACM Press, 2010.

J. Lengler, D. Sudholt, and C. Witt. Medium step sizes are harmful for the compact genetic algorithm. In Proc. of GECCO '18, pages 1499-1506. ACM Press, 2018.

F. G. Lobo, D. E. Goldberg, and M. Pelikan. Time complexity of genetic algorithms on exponentially scaled problems. In Proc. of GECCO '00, pages 151-158. Morgan Kaufmann, 2000.

H. Mühlenbein. How genetic algorithms really work: Mutation and hillclimbing. In Proc. of PPSN '92, pages 15-26. North Holland, 1992.
REFERENCES VII

H. Mühlenbein and T. Mahnig. Convergence theory and applications of the factorized distribution algorithm. Journal of Computing and Information Technology, 7:19-32, 1999.

H. Mühlenbein and T. Mahnig. FDA - A scalable evolutionary algorithm for the optimization of additively decomposed functions. Evolutionary Computation, 7(4):353-376, 1999.

F. Neumann, D. Sudholt, and C. Witt. A few ants are enough: ACO with iteration-best update. In Proc. of GECCO '10, pages 63-70. ACM Press, 2010

M. Pelikan and $\mathrm{H}$. Mühlenbein. The bivariate marginal distribution algorithm. In Advances in Soft Computing, pages 521-535. Springer, 1999.

M. Pelikan, K. Sastry, and E. Cantú-Paz. Scalable Optimization via Probabilistic Modeling: From Algorithms to Applications, volume 33 of Studies in Computational Intelligence. Springer, 2006.

\section{REFERENCES VIII}

M. Pelikan, M. Hauschild, and F. G. Lobo. Estimation of distribution algorithms. In Kacprzyk and Pedrycz (2015), pages 899-928.

G. Rudolph. Convergence properties of evolutionary algorithms. Verlag Dr. Kovač, 1997.

J. L. Shapiro. The sensitivity of PBIL to its learning rate, and how detailed balance can remove it. In Proc. of FOGA '02, pages 115-132. Morgan Kaufmann, 2003

J. L. Shapiro. Drift and scaling in estimation of distribution algorithms. Evolutionary Computation, 13(1):99-123, 2005.

J. L. Shapiro. Diversity loss in general estimation of distribution algorithms. In Proc. of PPSN '06, pages 92-101. Springer, 2006.

T. Stützle and H. H. Hoos. MAX-MIN ant system. Future Generation Computer Systems, 16(8):889-914, 2000

D. Sudholt and C. Thyssen. A simple ant colony optimizer for stochastic shortest path problems. Algorithmica, 64(4):643-672, 2012. ISSN 1432-0541.

REFERENCES IX

D. Sudholt and C. W. Update strength in EDAs and ACO: How to avoid genetic drift. In Proc. of GECCO '16, pages 61-68. ACM Press, 2016.

D. Thierens, D. Goldberg, and A. Pereira. Domino convergence, drift, and the temporal-salience structure of problems. In Proceedings of CEC '98, pages 535-540. IEEE Press, 1998.

C. W. Tight bounds on the optimization time of a randomized search heuristic on linear functions. Combinatorics, Probability and Computing, 22(2):294-318, 2013.

C. W. Upper bounds on the runtime of the univariate marginal distribution algorithm on OneMax. In Proc. of GECCO '17, pages 1415-1422. ACM Press, 2017.

C. W. Domino convergence: Why one should hill-climb on linear functions. In Proc. of GECCO '18, pages 1539-1546. ACM Press, 2018. 
REFERENCES X

Z. Wu and M. Kolonko. Asymptotic properties of a generalized cross-entropy optimization algorithm. IEEE Transactions on Evolutionary Computation, 18(5):658-673, 2014.

Z. Wu, M. Kolonko, and R. H. Möhring. Stochastic runtime analysis of the cross-entropy algorithm. IEEE Transactions on Evolutionary Computation, 21(4):616-628, 2017.

Q. Zhang. On the convergence of a factorized distribution algorithm with truncation selection. Complexity, 9(4):17-23, 2004a.

Q. Zhang. On stability of fixed points of limit models of univariate marginal distribution algorithm and factorized distribution algorithm.

IEEE Transactions on Evolutionary Computation, 8(1):80-93, 2004b.

Q. Zhang and H. Mühlenbein. On the convergence of a class of estimation of distribution algorithms. IEEE Transactions on Evolutionary Computation, 8(2):127-136, 2004. 